\title{
The Impact of Voucher Coupons on the Uptake of Fertilizer ANd IMProved SEeds: EvidenCE From A Randomized Trial in MozambiQue
}

\author{
Michael R. Carter, Rachid LaAjaj, and Dean Yang
}

The use of improved seeds and fertilizer contributed to large productivity gains in many parts of the developing world over the last 50 years. And yet this green revolution largely bypassed the African continent, which, over the 1960 to 2000 period, registered the lowest yield increases of all world regions (Evenson and Gollin 2003). According to Food and Agriculture Organization of the United Nations (FAO) statistics, African farmers in 2009 used on average only 13 kilograms of fertilizer per hectare, compared with an average of 94 kilograms per hectare in other developing countries.

In response to this apparent underutilization of fertilizers, a number of African governments have recently introduced fertilizer subsidies that operate through voucher coupons. Despite the widespread adoption of this instrument, the effectiveness of voucher coupons in boosting input use in both the short and the medium term remains largely untested. ${ }^{1}$ In an effort to fill this evidence gap, we here report initial results from a pilot intervention in Mozambique designed to evaluate the impact of voucher coupons in both the short and medium terms.

\footnotetext{
Michael Carter (mrcarter@ucdavis.edu) is Professor, Department of Agricultural and Resource Economics, University of California, Davis. Rachid Laajaj (rachid.laajaj@parisschoolofeconomics.eu) is a Post-doctoral Fellow, Paris School of Economics and INRA. Dean Yang (deanyang@umich.edu) is Professor, Ford School of Public Policy, University of Michigan. We gratefully acknowledge financial support from the U.S. Agency for International Development Cooperative Agreement No. EDH-A-00-06-0003-00 through the BASIS Assets and Market Access CRSP. We also thank the International Fertilizer Development Center and the Banco Oportunidade de Mocambique for their support and collaboration in the implementation of this work.

This article was presented in an invited paper session at the 2013 ASSA annual meeting in San Diego, CA. The articles in these sessions are not subjected to the journal's standard refereeing process.

${ }^{1}$ There is also debate about whether fertilizer use is even profitable in many parts of Africa.
}

Unlike many of its neighbors that launched nationwide fertilizer subsidy programs, Mozambique piloted a limited, two-year fertilizer subsidy program for maize and rice producers. The pilot targeted 25,000 farmers over the 2009-10 and 2010-11 crop years. The limited scope of this program allowed the research team, in cooperation with the Ministry of Agriculture in Mozambique, to design and implement a randomized controlled trial of the voucher coupon system. In addition, the research team overlaid the voucher intervention with a savings promotion program designed to see if improved savings services would enhance the long-run impacts of the voucher program.

In this paper, we lay out the logic and impact evaluation methodology of this multidimensional intervention. We also report first results showing the short-term impacts of the voucher program on farmer use of improved seeds and fertilizer. Using methods that account for the endogenous decision to use randomly distributed voucher coupons, we find that impact of the voucher coupons on use of seeds and fertilizers is large for those that choose to use them. While this finding indicates that there are real liquidity and other constraints that block the use of the inputs by small-scale farmers, we also puzzlingly find that the uptake and use of the voucher coupons is surprisingly low, well below $50 \%$. These latter findings signal the existence of additional constraints that must be addressed if higher uptake rates are to be obtained even in the short run.

\section{Short-Term Logic and Limitations of Voucher Coupons}

In the 1970s and early 1980s, a majority of African countries subsidized and sold fertilizer 
through state-owned enterprises. These programs were roundly criticized for being costly, inefficient, overwhelmingly beneficial to large farmers, and detrimental to the private sector. In contrast, voucher coupons are intended for distribution exclusively to poor farmers, who then use the coupons to purchase inputs, often with a cash co-payment. These voucher schemes have been argued to offer the advantages of traditional fertilizer subsidy, while targeting the poor more effectively and stimulating, rather than undermining, the private sector.

In principle, these voucher schemes should address the many barriers traditionally hypothesized to limit the uptake of improved technology by resource-constrained farmers, including credit constraints, risk aversion, information barriers on how to use the technology, information on returns to the technology, and constraints to the reliable supply of agricultural inputs. Making inputs affordable during a limited period can allow farmers to experiment and familiarize themselves with the technology, while making a profit that will allow them to save enough to afford further inputs in the future. Under this logic, coupons will stimulate reliable, long-term demand for seeds and fertilizer, which in turn is expected to stimulate a reliable and widely spread supply of these inputs by the private sector.

While this short-term logic of voucher coupons is straightforward, it rests on a multiplicity of assumptions beyond the presumed profitability of fertilizers and improved seeds. These assumptions include the government's ability to efficiently target and administer the program, the responsiveness of private input suppliers to voucher-induced demand, and small farmers' willingness and ability to meet co-payments and to learn rapidly despite the stochasticity of production. There is some evidence that these assumptions fail. Minot and Benson (2009), for example, document instances in which voucher programs have failed to really target the poor and generally exhibited low cost effectiveness.
The randomized trial reported here is intended to speak directly to these short-term issues. Table 1 illustrates the experimental design for our study. Along the first dimension (the rows of table 1), a set of vouchereligible farmers was randomly divided between those that gained access to voucher coupons (we term this group "voucher winners") and those that did not ("voucher losers"). The latter group can of course function as a valid control group for the former. The short-run question is whether input use and ultimately yields increase over the two-year pilot period for those that gain access to the voucher coupons.

\section{Medium-Term Logic and Limitations of Voucher Coupons}

While voucher coupons may be effective in the short run of the subsidy, a second question is what happens after the expiration of the voucher program. While some countries (e.g., Malawi) have made voucher coupons a permanent (and expensive) feature of agricultural policy, others presume that self or other sources of finance can replace the subsidies provided by the coupons and sustain the uptake of the improved technologies. While yield increases might be sustained, it may also be the case that input use and yields return to the level of the control group that never experienced the subsidies. In Mozambique, where small farm households rarely have institutionalized savings, and where small farm agricultural credit is almost nonexistent, the question about the sustainability of technology uptake is especially relevant.

As part of a long-term research strategy, the randomized trial set up to explore the impacts of voucher coupons in Mozambique was expanded to consider the impact of savings interventions on the sustainability of any technology uptake induced by the voucher coupons. In collaboration with the Opportunity Bank of Mozambique's mobile banking services, the research team designed several savings interventions (ranging from simple

Table 1. Treatment Regimes and Number of Observations

\begin{tabular}{lccc}
\hline & $\begin{array}{c}\text { No Savings } \\
\text { (Education } \\
\text { Session } \\
\text { Only) }\end{array}$ & $\begin{array}{c}\text { Savings Account } \\
\text { Encouraged } \\
\text { (Information and } \\
\text { Financial Education) }\end{array}$ & $\begin{array}{c}\text { Savings Account } \\
\text { Encouraged + } \\
\text { Matched Savings }\end{array}$ \\
\hline Voucher winners (795) & $\mathrm{R}_{00}(267)$ & $\mathrm{R}_{01}(283)$ & $\mathrm{R}_{03}(245)$ \\
Voucher losers (798) & $\mathrm{R}_{10}(247)$ & $\mathrm{R}_{11}(311)$ & $\mathrm{R}_{23}(240)$ \\
\hline
\end{tabular}


education to a matched savings program designed to further incentivize use of formal savings instruments).

As illustrated in table 1, both voucher winners and losers were randomly divided among three savings intervention groups. The base group was simply informed about the availability of mobile banking, the second group was given additional help on account opening and financial training on the advantages of institutionalized savings, while a third group was also given "matched savings" incentives (meaning individuals received a $50 \%$ savings match if they met a prespecified savings target calibrated on the costs of a maize input package). Over the longer term, analysis of the technology use of these different groups should help signal which constraints limit the uptake of improved seeds and fertilizers. The remainder of this paper, however, will focus only on the short-term voucher coupon impacts of the pilot.

\section{The Voucher Coupon Pilot in Mozambique}

Agriculture in Mozambique is dominated by small-scale farming, with little to no use of tractors, plows, fertilizer, pesticides, irrigation, and other agro-inputs. Small farms use fertilizer, if at all, on cash crops rather than on cereals. Yields of the latter are low, generally below 1 ton per hectare for maize production (compared to up to 8 tons per hectare in the most productive developing countries). The nascent input market is small and its network unsubstantial.

Against this backdrop, Mozambique launched its two-year voucher coupon pilot intended to benefit 25,000 small-scale maize and rice producers. ${ }^{2}$ The maize portion of the program benefited 15,000 farmers in 3 provinces, with 5000 of them in Mancia province, which is in the center west of the country and shares a border with Zimbabwe. For maize, the voucher coupon underwrote the purchase of a technology package designed for a half hectare of improved maize production: $12.5 \mathrm{~kg}$ of improved seeds (either OPV or hybrid) and $100 \mathrm{~kg}$ of fertilizer $(50 \mathrm{~kg}$ of urea and $50 \mathrm{~kg}$ of NPK 12-24-12). The market value

\footnotetext{
2 The European Union funded the pilot, which was implemented by the Food and Agriculture Organization and the International Fertilizer Development Center in collaboration with the Ministry of Agriculture in Mozambique.
}

of this package was about USD 117, with farmers required to co-pay about USD 32, or $27 \%$ of the total cost.

For purposes of this study, 75 villages in Manica were selected. Within villages, individuals were deemed eligible for a voucher coupon if they met the standard program criteria:

- Farming between 0.5 hectare and 5 hectares of maize;

- Being a "progressive farmer," defined as a producer interested in modernization of their production methods and commercial farming;

- Having access to agricultural extension and access to input and output markets;

- Being able and willing to pay for the remaining $27 \%$ of the package cost.

Given the absence of prior data on maize cultivated and other necessary information, lists of eligible farmers were created jointly by agricultural extension, local leaders, and agroinput retailers, under the supervision of the International Fertilizer Development Center. Farmers were asked to register only if they had the money to complete the subsidy. Only one person per household was allowed to register. The farmers were informed that a lottery would occur and only half of those on the list would win a voucher. After official approval of the Provincial Service of Agriculture of Manica, the lists of possible participants were used to randomly assign vouchers to $50 \%$ of the households in the list of each village. After winning the voucher lottery, individuals still had to decide whether to pick up the voucher, as well as whether and how to use it. Villages and individuals were subsequently divided among the different savings treatment, resulting in the final sample sizes shown in table 1.

Baseline statistics on the sample corroborate the validity of the randomization as there are no significant differences between voucher winners and losers in either household or farm characteristics (see online appendix table A-1). Sample household heads are $84 \%$ male and $76 \%$ are literate. By comparison, in rural Manica province, only $66 \%$ of household heads are male and $45 \%$ are literate, an indication that the targeted households are relatively less vulnerable compared to the rest of the region. Study households own an average 10.3 hectares of land owned (the median is 5 hectares). Eleven percent of households have electricity at home, and $19 \%$ used fertilizer on at least one 
Table 2. Intention to Treat Impact Estimates

\begin{tabular}{lcccccc}
\hline & \multicolumn{2}{c}{$\begin{array}{c}\text { Fertilizer } \\
(\mathrm{kg})\end{array}$} & $\begin{array}{c}\text { Improved Seeds } \\
(\mathrm{kg})\end{array}$ & $\begin{array}{c}\text { Voucher } \\
\text { Received }\end{array}$ & $\begin{array}{c}\text { Voucher } \\
\text { Used }\end{array}$ \\
\hline Won lottery & $11.6^{*}$ & $14.8^{* * *}$ & 2.7 & $3.1^{* *}$ & $0.38^{* * *}$ & $0.22^{* * *}$ \\
& {$[5.85]$} & {$[2.20]$} & {$[1.87]$} & {$[1.27]$} & {$[0.02]$} & {$[0.02]$} \\
Fertilizer prior season $(\mathrm{kg})$ & $2.0^{* * *}$ & $0.8^{* * *}$ & & & & \\
& {$[0.61]$} & {$[0.13]$} & & & & \\
Improved seeds & $-3.5^{*}$ & 0.3 & -0.01 & 0.02 & & \\
Prior season (kg) & {$[2.05]$} & {$[0.42]$} & {$[0.38]$} & {$[0.31]$} & & \\
Maize area & & & $0.8^{* * *}$ & $0.9^{* * *}$ & & \\
Prior season (ha) & & & {$[0.06]$} & {$[0.05]$} & & $\mathbf{0 . 1 2}]$ \\
Constant & -7.3 & 2.7 & 3.0 & 1.7 & $\mathbf{0 . 0 6}$ \\
& {$[10.27]$} & {$[3.30]$} & {$[1.87]$} & {$[1.44]$} & {$[\mathbf{0 . 0 1}]$} & {$[\mathbf{0 . 0 1}]$} \\
Observations & 1,408 & 1,393 & 1,414 & 1,400 & 1,429 & 1,436 \\
R-squared & 0.70 & 0.54 & 0.61 & 0.76 & 0.17 & 0.08 \\
Trimming & $0 \%$ & $1 \%$ & $0 \%$ & $1 \%$ & $0 \%$ & $0 \%$ \\
\hline
\end{tabular}

Note: Robust standard errors in brackets; ${ }^{* * *} \mathrm{p}<0.01,{ }^{* *} \mathrm{p}<0.05,{ }^{*} \mathrm{p}<0.1$

All regressions include village fixed effects.

Trimming removes observations with highest absolute deviation between current and previous period

of their maize fields during the preintervention 2009-2010 season. While better off than some, the study population is dominated by relatively poor small-scale farmers with limited experience with modern agro-inputs.

\section{The Impact of Voucher Coupons on the Use of Fertilizer and Improved Seeds}

This section investigates the impact of voucher coupons on the use of fertilizer and improved seeds. In addition to baseline data, the analysis here relies on data covering the second year of the voucher program, 2010-2011. A third survey round completed in the latter half of 2012 will allow inference on the effectiveness of the savings interventions.

As described in the preceding section, voucher lottery winners had to pick up and then decide whether and how to use the voucher coupons. To examine impacts, we will first look at the impact of simply winning the voucher lottery on seed and fertilizer use. After estimating these intention to treat effects, we consider some of the factors that limited the pickup and use of voucher coupons. Using the voucher lottery results as an instrument, we also estimate local average treatment by looking at the impacts of voucher receipt and voucher use on improved seed and fertilizer uptake.

\section{Intention to Treat Estimates}

The randomization of the voucher lottery allows us to exploit the following simple reduced form equation to obtain intention to treat estimates of the impact of voucher coupons on seed and fertilizer use:

$$
Y_{i v}=a_{0}+a_{1} Z_{i v}+X_{i v}^{\prime} a_{2}+\theta_{i v}+\varepsilon_{i v}
$$

where $Y_{i v}$ is either seed or fertilizer use by household $i$ in village $v, Z_{i v}$ is an indicator equal to 1 if individual $i$ was assigned to the treatment group (i.e., won the voucher lottery) and 0 otherwise, $\theta_{v}$ is a village fixed effect, $X_{i v}$ is a vector of control variables (which generally includes the outcome at the previous period and the maize area cultivated), and $\varepsilon_{i v}$ is a mean zero error term of household $i$. Since the randomization of the treatment ensures that $Z_{i v}$ and $\varepsilon_{i v}$ are orthogonal, the ordinary least squares provide an unbiased estimator of $a_{1}$, the Intention to Treat (ITT) effect. Village fixed effects and control variables are not necessary to obtain unbiased results but increase the precision of the estimator.

Table 2 reports these ITT effects for both fertilizer use and use of improved seeds. Both variables are measured as the total kilograms of both products used for maize cultivation. Results are presented for both the full sample, as well as for a trimmed sample, which eliminated the $1 \%$ of observations that showed the largest change in the dependent variable relative to baseline.

As can be seen, the ITT estimates are statistically significant, but the magnitudes are modest as they show that compared to the control group, the treatment group used on 
average 14.8 additional kilograms of fertilizer and $3.1 \mathrm{~kg}$ of improved seeds (using the trimmed results). As the voucher package subsidized the purchase of 100 kilograms of fertilizer and 12.5 kilograms of improved seeds, these estimates indicate substantial slippage between program intent and program impact.

The last two columns of table 2 give a first indication of what underlies these modest ITT estimates. Reported in these columns are the results from two regressions. The first regresses a binary indicator of whether the household picked up and actually received a voucher coupon on the lottery outcome variable. The second regresses an indicator of whether the household cashed in and used the voucher coupon for its maize production on that same explanatory variable. As shown in the table, winning the lottery increased the probability of receiving a voucher from $12 \%$ to $50 \%$, and it increased the chances of cashing in and using a voucher for maize production from $6 \%$ to $28 \%$.

These estimates reveal that there was significant leakage of vouchers to the control group. These leakages came from two sources. First, some lottery losers tried to negotiate a voucher from the government's extension service. The extension service was charged with redistributing all unused vouchers to other households. While this redistribution was to take place outside the study area in order not to contaminate the sample, it is likely that in some cases unused vouchers were distributed to lottery losers.

Second, we can see that the ITT estimates are diluted by the low voucher pickup and use rates by lottery winners. When entitled to receive a voucher, only half of beneficiaries picked it up and, conditional on picking up the voucher, only $57 \%$ redeemed it and used the content of the package for their maize production.

\section{Local Average Treatment Effects of the Voucher Program}

In an effort to understand these modest uptake rates, our survey queried individuals directly about their decisions to pick up and use voucher coupons. Almost half of the farmers $(46 \%)$ who did not pick up a voucher despite winning the lottery indicated that they lacked the USD 32 co-payment required to use the voucher. ${ }^{3}$ Other farmers mentioned not

\footnotetext{
${ }^{3}$ Extension workers stressed to farmers that they should not pick up the voucher if they lacked the funds to use it (so that the voucher could be given to another person who could use it).
}

being present at the time of voucher distribution (17\% of cases) or the late distribution of vouchers (16\% of cases) as the main reasons for not picking up their vouchers. Among those who picked up and received a voucher, $83 \%$ redeemed it, and $57 \%$ used the fertilizer obtained by the voucher for their maize production.

Among those who received the voucher but did not redeem it, $54 \%$ reported not having the necessary amount of money to complete the subsidy, and $36 \%$ reported the nonavailability or late arrival of the agro-inputs at the agrodealer or the distance to the closest agro-dealer as reasons for nonparticipation. Among those who redeemed the voucher but did not use the fertilizer for maize production, $67 \%$ reported using it on other agricultural production (e.g., tobacco, horticulture), $25 \%$ claimed they had not yet used it, and $4 \%$ stated that they sold their fertilizer.

These responses reveal the complexity of voucher programs. They also consistently signal the importance of liquidity constraints in determining who actually takes up voucher coupons, at least when co-payments are required. While the ITT estimates in the prior section accurately identify the average impact on the target population, they understate the overall impact of the voucher program given that unused vouchers were redistributed to other farmers. In order to estimate this overall program impact, we implemented the following instrumental strategy to estimate the vouchers on the input use of those who chose to receive and use them:

$$
Y_{i v}=b_{0}+b_{1} \hat{D}_{i v}+X_{i v}^{\prime} b_{2}+\theta_{v}+\varepsilon_{i v}
$$

where $\hat{D}_{i v}$ is the fitted value of voucher received (voucher used) from the regressions estimated in table 2, which use the random variable "won lottery" as an instrument.

Table 3 displays the estimated increase in input use conditional on using the voucher (online appendix table A-2 displays the results for picking up the voucher). Compared to the ITT estimate that winning the lottery boosted fertilizer use by 14 kilograms, the instrumental variable estimate of the impact of receiving the coupon is 39 kilograms, while the estimated impact of using the coupon for maize is 68 kilograms (table 3 ). This latter figure is still short of the 100 kilograms of fertilizer subsidized by the coupon, indicating that some farmers only used part of the package or were simply substituting 
Table 3. IV Impact Estimates of Using Voucher

\begin{tabular}{|c|c|c|c|c|}
\hline \multirow{3}{*}{$\begin{array}{l}\text { Voucher used } \\
\quad \text { (instru- } \\
\text { mented) }\end{array}$} & \multicolumn{2}{|c|}{$\begin{array}{l}\text { Fertilizer } \\
\quad(\mathrm{kg})\end{array}$} & \multicolumn{2}{|c|}{$\begin{array}{l}\text { Improved Seeds } \\
(\mathrm{kg})\end{array}$} \\
\hline & $52.2^{* *}$ & $68.3^{* * *}$ & 12.1 & $14.4^{* * *}$ \\
\hline & [26.54] & {$[9.34]$} & [7.43] & {$[5.40]$} \\
\hline \multirow{2}{*}{$\begin{array}{l}\text { Fertilizer } \\
\quad \text { prior } \\
\quad \text { season }(\mathrm{kg})\end{array}$} & $2.0^{* * *}$ & $0.7^{* * *}$ & & \\
\hline & {$[0.61]$} & {$[0.12]$} & & \\
\hline $\begin{array}{l}\text { Improved } \\
\text { seeds }\end{array}$ & -3.3 & 0.6 & 0.03 & 0.07 \\
\hline $\begin{array}{l}\text { Prior season } \\
\quad(\mathrm{kg})\end{array}$ & [2.14] & {$[0.40]$} & {$[0.30]$} & {$[0.25]$} \\
\hline Maize area & & & $0.8^{* * *}$ & $0.8^{* * *}$ \\
\hline $\begin{array}{l}\text { Prior season } \\
\text { (ha) }\end{array}$ & & & {$[0.07]$} & {$[0.05]$} \\
\hline Observations & 1,403 & 1,388 & 1,410 & 1,397 \\
\hline R-squared & 0.70 & 0.64 & 0.61 & 0.75 \\
\hline Trimming & $0 \%$ & $1 \%$ & $0 \%$ & $1 \%$ \\
\hline
\end{tabular}

Note: See table 2 notes.

for fertilizer that they would have purchased even without the coupon.

The results for improved seeds are somewhat stronger. Recalling that the coupon subsidized the purchase of 12.5 kilograms of improved seed, we see that the IV estimate for those who picked up and used the coupon for their maize production is 14.4 kilograms (table 3 ).

Keeping in mind that these results could have been zero (had farmers simply been replacing their own self-financed purchases with the subsidized packages, or if they had simply been selling off the inputs to largerscale farmers), this analysis shows that there are indeed strong constraints to the adoption of improved seeds and fertilizers among Mozambique's small-scale maize farmers. At the same time, the modest use rates of the coupons signal difficulty of broadening the use of modern inputs among a poor population with little prior experience in using it.

\section{Conclusions}

We have reported here results from a study undertaken in collaboration with the government of Mozambique in which we randomly selected $50 \%$ of a sample of small-scale maize farmers to receive voucher coupons that entitled them to receive a $73 \%$ subsidy on a package comprised of 100 kilograms of fertilizer and 12.5 kilograms of improved maize seeds. Intention to treat estimates reveal that the farmers selected to receive the coupons used 15 kilograms more fertilizer and 3 kilograms more improved seeds than did the control group. Instrumental variable estimates of the impact of actually using the voucher reveal impacts of 68 kilograms of fertilizer and 14 kilograms of maize seed. The difference between the two estimates reflects the relatively low rate of uptake and use of coupons by those selected to receive them.

This relatively low use or compliance rate hints at the existence of other constraints (e.g., liquidity and informational) that limit the uptake of higher yield production practices. Even among those who used the subsidy, two important questions remain. First, did the agro-input package increase yields and family income, and if so, did it do so in a uniform fashion or is there strong impact heterogeneity? Second, assuming that the voucher increases incomes for those who used it, were they able to sustain their use of the improved technological package once the voucher program ended? As discussed above, a second dimension of the intervention studies examines this question and the degree to which a series of complementary savings interventions may have shaped the sustainability of the technology uptake.

Work examining both questions is currently underway. Preliminary results suggest that the yield and income results are quite heterogeneous and that the matched savings intervention led to large increases in formal savings (Carter, Laajaj, and Yang 2013). Interestingly, as discussed in Laajaj (2012), these interventions seemed to have had a significant impact on individual's planning time horizons, with the poorer beneficiaries of these programs showing substantially greater patience than those randomly selected not to receive the program. Future research will also explore whether these increased time horizons spill over and result in increased saving and technology adoption in the future.

\section{Supplementary Material}

Supplementary material is available at the American Journal of Agricultural Economics online at www.oxfordjournals.org/our_ journals/ajae. 


\section{References}

Carter, M.R., R. Laajaj, and D. Yang. 2013. The Heterogeneous Impact of Agro-Input Subsidies on Maize Production: A Field Experiment in Mozambique. 2013. Working Paper, Dept. of Agr. Econ., University of California, Davis.

Evenson, R.E., and D. Gollin. 2003. Assessing the Impact of the Green Revolution, 1960 to 2000. Science 300(5620):758-62.
Laajaj, R. 2012. Closing the Eyes on a Gloomy Future: Psychological Causes and Economic Consequences. Working Paper, Paris School of Economics.

Minot, N., and T. Benson. 2009. Fertilizer Subsidies in Africa: Are Vouchers the Answer? Technical Report Brief No. 60, International Food Policy Research Institute (IFPRI), Washington, DC. 